\title{
Keine Angst vor der Digitalisierung! Zum Stand digitalisierter Arbeitsanforderungen in verschiedenen Industriebranchen und Tätigkeitsfeldern sowie Zusammenhänge zwischen Belastung, Ressourcen und Beanspruchungsfolgen in Deutschland
}

\author{
Christian Härtwig ${ }^{1,2}$ - Anna Sapronova ${ }^{2}$
}

Online publiziert: 23. April 2020

(c) Der/die Autor(en) 2020

\section{Zusammenfassung}

Ziel der Studie war es, den Stand der Digitalisierung in zwölf Industriebranchen in Deutschland zu untersuchen. In der Fragebogen-Erhebung unter 14.007 Beschäftigten zeigte sich, dass die Digitalisierung oft noch am Anfang steht und primär durch den Einsatz von IKT-Systemen geprägt ist. Unterschiede zwischen acht verschiedenen Blue- und White-Collar-Tätigkeitsfeldern fallen statistisch bedeutsamer aus als zwischen einzelnen Branchen in Hinblick auf die Nutzung digitaler Arbeitsmittel, Arbeitsanforderungen und Ressourcen. Überraschenderweise zeigt sich eine hohe Bereitschaft und Zuversicht der Beschäftigten, die Veränderungen der Digitalisierung zu bewältigen; Befürchtungen und Unsicherheiten spielen in der Breite der Belegschaften eine eher untergeordnete Rolle. Anhand eines Strukturgleichungsmodells werden Wirkzusammenhänge zwischen allgemeinen sowie digitalisierungsspezifischen Arbeitsanforderungen und Ressourcen, Aspekten der beruflich-sozialen Entkopplung und psychischen Beanspruchungsfolgen deutlich. Mögliche Ergänzungen theoretischer Modelle werden diskutiert sowie Anregungen für Forschung und betriebliche Praxis skizziert.

Praktische Relevanz: In diesem Beitrag werden Ansätze zur Regulierung allgemeiner und digitalisierungsspezifischer Belastung sowie Stärkung arbeitsbezogener und persönlicher digitaler Ressourcen aufgezeigt. Dabei sollten Besonderheiten verschiedener Tätigkeitsfelder stärker berücksichtigt werden als Unterschiede zwischen verschiedenen Branchen. Trotz grundsätzlich positiver Befunde wird vorgeschlagen, Tendenzen beruflich-sozialer Entkopplung frühzeitig entgegenzuwirken.

Schlüsselwörter Digitalisierung · White Collar und Blue Collar Tätigkeitsfelder · Industriebranchen · Chemische Industrie · Digitale Selbstwirksamkeit · Unterschiedseffekte · Beruflich-soziale Entkopplung · Strukturgleichungsmodell

Prof. Dr. Christian Härtwig

christian.haertwig@fom.de

1 Institut für Wirtschaftspsychologie, FOM Hochschule für Ökonomie \& Management gGmbH, Berlin, Deutschland

2 Goodwork GmbH, Berlin, Deutschland 


\title{
Don't be afraid of digitization! On the status of digitized work requirements in different industrial sectors and fields of work as well as interactions between job demands, resources and strain reactions in Germany
}

\begin{abstract}
The aim of this study was to examine the status of digitization in twelve industrial sectors in Germany. Results of the questionnaire survey of 14,007 employees show that digitization is still in its infancy and is primarily characterized by the use of ICT systems. Regarding the use of digital work equipment, as well as job demands and resources, differences between eight blue- and white-collar jobs are statistically more relevant than differences between industrial sectors with regard to the use of digital work equipment, job demands and resources. Surprisingly, employees displayed high levels of willingness and confidence to cope with the changes in digitization; fears and uncertainties play a rather subordinate role in the breadth of the workforces. Structural equation modeling elucidated cause-effect relationships between general and digitization-specific job demands and resources as well as aspects of occupational-social decoupling and consequences of psychological stress. Potential expansions of existing theoretical models are discussed and suggestions for future research as well as operational practice are outlined.

Practical Relevance: This article proposes starting points for the regulation of general and digitization-specific job demands as well as the strengthening of work-related and personal digital resources. The particularities of different fields of work should be taken into account more than supposed differences between industrial sectors. Despite these overall positive findings, the authors advise to counteract tendencies towards occupational-social decoupling at an early stage.
\end{abstract}

Keywords Digitization - White Collar and Blue Collar jobs · Industrial sectors · Chemical industry · Digital self-efficacy $\cdot$ Difference effects $\cdot$ Occupational-social decoupling $\cdot$ Structural equation model

\section{Einleitung}

Die Digitalisierung verändert die beruflichen Tätigkeiten und Rahmenbedingungen quer durch alle Branchen für eine Großzahl von Beschäftigten (Hermeier et al. 2019). Auch in den Industriebranchen ergeben sich trotz bereits fortgeschritten-automatisierter Arbeitsprozesse zusätzliche und ganz neue Anforderungen (Hämmerle et al. 2017; Priddat und West 2016). So sind in digitalisierten Arbeits- und Produktionsprozessen Beschäftigte, Maschinen, Produkte und Prozesse miteinander verknüpft und tauschen Informationen in Echtzeit aus. Autonome Systeme kommunizieren untereinander und führen selbstständig Koordinationsund Entscheidungsprozesse durch. Mit neuer Sensorik und Kommunikation entstehen neue Formen der Zusammenarbeit zwischen Menschen und kollaborativen Robotersystemen. Der Mensch entwickelt sich an vielen Stellen verstärkt hin zum Problemlöser, Entscheider und Innovator.

In öffentlichen Debatten wird daher diskutiert, welchen Effekt die Einführung dieser neuen digitalen Technologien auf die Beschäftigten und die Arbeit selbst hat. In der arbeitswissenschaftlichen Literatur wird angenommen, dass sich sowohl die Belastung wandelt (z. B. durch zunehmende Vernetzung, Arbeitsgeschwindigkeit und zeitlich-örtliche Unabhängigkeit), als auch die Arbeitsgestaltung, Arbeitsorganisation, Zusammenarbeit, Koordination und Führung bei arbeitenden Menschen, Gruppen und Organisationen sowie ganzen Branchen (Hirsch-Kreinsen et al. 2018).
Spezifische Studien zu Art und Umfang dieser Veränderungen fehlen allerdings bisher.

Infolge der neuen Anforderungen und betrieblichen Restrukturierungen wird angenommen, dass sich v.a. die psychische Belastung, aber auch die resultierende Beanspruchung und Beanspruchungsfolgen der Beschäftigten intensivieren (Hasselmann et al. 2017); auch zunehmende Entfremdung und Distanzierung der Beschäftigten zum bisher vertrauten und sich schrittweise digitalisierenden Beruf und Betrieb sowie zunehmende berufliche Unsicherheiten sind denkbar (Maier et al. 2017). Angenommen werden aber auch positive Effekte im Sinne fortschreitender Persönlichkeitsförderlichkeit durch die Weiterentwicklung von Tätigkeiten mit der Entwicklung neuer Motivations-, Wachstums- und Leistungspotenziale. Es herrscht eine konstante Unsicherheit in Hinblick auf das Ausmaß und die Qualität dieser Veränderungen. So existieren unterschiedliche Befunde zur Auswirkung einer arbeitsbezogenen Nutzung digitaler Informations- und Kommunikationstechnologien auf psychische Gesundheit und Work-Life-Balance: Einerseits werden bessere Flexibilität und Vereinbarkeit von Berufsund Privatleben (Leung 2011) angepriesen, andererseits eine zunehmende psychische Irritation durch ständige Erreichbarkeit und das Verschwimmen der Grenzen zwischen von Berufs- und Privatleben kritisiert (Ninaus et al. 2015). Arbeitsbezogene Faktoren können die Zusammenhänge sowohl positiv, als auch negativ wirken. In der vorliegenden Studie möchten wir Faktoren untersuchen, die diesen $\mathrm{Zu}$ sammenhang im Kontext der Digitalisierung erklären kön- 
nen, um in der Praxis negative Effekt von Digitalisierung abzufedern und wirksame Ressourcen zu stärken.

Zudem stellt sich die Frage, welchen Einfluss allgemeine berufliche Rahmenfaktoren auf die Belastung und Beanspruchung im Zuge der Digitalisierung haben. In der Literatur werden sowohl Branchen-, als auch Tätigkeitsunterschiede diskutiert - mitunter werden die beiden Ebenen auch miteinander vermischt. Belastbare empirische Befunde z.B. für Unterschiede zwischen spezifischen Industriebranchen zu den Entwicklungen der neuen Arbeitswelt sowie zum Effekt des Wandels auf die Beschäftigten fehlen allerdings (Absenger et al. 2016). Aufgrund der geringen Anzahl von Studien, die Digitalisierung und ihre Einflüsse auf Arbeitnehmende systematisch untersuchen, wurde in der vorliegenden deutschlandweiten Studie $\mathrm{Mo}$ nitor Digitalisierung ${ }^{1}$ explorativ betrachtet, wie sich die Digitalisierung derzeit im beruflichen Alltag bei Beschäftigten darstellt. Es wurde untersucht, welche Technologien genutzt werden, welche Auswirkungen der Digitalisierung sich in verschiedenen Industriebranchen und Tätigkeitsfeldern zeigen und inwiefern es hier Unterschiede gibt. Anhand von Daten der Chemiebranche wurde untersucht, welche Zusammenhänge und Wirkfaktoren zum Verständnis der Belastungs-Ressourcen-Beanspruchungs-Konstellationen in digitalen Kontexten beitragen. Die gewonnenen Erkenntnisse sollen dazu beitragen, relevante Einflussfaktoren und Wirkzusammenhänge zu identifizieren, um spezifische Unterstützungsmaßnahmen und abgestimmte Hilfsprogramme für Betriebe im digitalen Wandel zu entwickeln.

\section{Theoretischer und empirischer Hintergrund}

Die Digitalisierung ist ein zunehmendes und umfangreiches Phänomen im beruflichen Alltag und beeinflusst Unternehmen, deren Beschäftigte und ihr Wohlbefinden bei der Arbeit. Im Jahr 2018 schätzten knapp die Hälfte der Unternehmen die Digitalisierung als sehr wichtig ein, zehn Prozent mehr als im Jahr 2016 (BMWi 2018). Angesichts des schnellen technologischen Fortschritts und der zunehmenden Integration von Digitalisierung in den Arbeitskontext zeigen Studien verschiedene Möglichkeiten und Auswirkungen der Digitalisierung, die komplexe Wirkungen und sowohl Vorteile, als auch Nachteile für die Beschäftigten und Betriebe mit sich bringen (Ninaus et al. 2015). Diskutiert werden verschiedene Entwicklungsrichtungen: eine intensivierte Automatisierung und Digitalisierung der Tätigkeiten, eine Aufwertung im Sinne neuer Anforderungen

\footnotetext{
${ }^{1}$ Die Studie wurde unterstuitzt von der Stiftung Arbeit und Umwelt der IG BCE.
}

(z.B. mit der Entwicklung und Förderung neuer Fähigkeiten und Fertigkeiten) oder keine Veränderungen. Insgesamt wird eine generelle Aufwertung von Tätigkeiten und Qualifikationen im Zuge einer allgemeinen Informatisierung der Arbeit erwartet (vgl. Hirsch-Kreinsen et al. 2018).

Zur Eingrenzung der in dieser Studie zentralen Aspekte der Belastung und Auswirkung der Digitalisierung wird zunächst als theoretischer Rahmen das klassische BelastungsBeanspruchungsmodell (Hackman und Oldham 1980) sowie das darauf aufbauende Job-Demands-Resources-Modell von Bakker und Demerouti $(2007,2014)$ herangezogen. Demnach nehmen sowohl Arbeitsanforderungen und allgemeine Belastung, als auch Arbeits- und persönliche Ressourcen sowie individuelle Voraussetzungen (z.B. Fähigkeiten und Einstellungen) Einfluss auf die kurzfristige Beanspruchung sowie auf längerfristige Beanspruchungsfolgen wie Gesundheit und Wohlbefinden der Beschäftigten. Für diesen theoretischen Rahmen liegen in Hinblick auf die Digitalisierung bereits einige Forschungsbefunde vor, die im Folgenden kurz skizziert werden:

Für die klassische Betrachtung von Belastung und Beanspruchung bzw. Beanspruchungsfolgen ist zunächst zu berücksichtigen, mit welchen digitalen Technologien die Beschäftigten arbeiten und mit welchen weiteren Belastungsaspekten diese einhergehen. Anhand einer Studie des BMAS (2016) konnte gezeigt werden, dass ein Großteil der Befragten (83\%) bereits digitale Technologien (hier vor allem i.S. von Informations- und Kommunikationstechnologien, kurz: IKT) am Arbeitsplatz nutzt, dabei aber deutliche Unterschiede zwischen Berufsgruppen sowie klare Zusammenhänge zwischen Nutzungsgrad und Höhe des Ausbildungsniveaus zu finden sind. Im Zuge der Nutzung von IKT wird von einem höheren Arbeitstempo, höherem Zeitdruck (Atanasoff und Venable 2017) sowie längeren Arbeitstagen berichtet (Sellberg und Susi 2014). Beschäftigte in neuen technisierten Arbeitsbedingungen würden auch eine stärkere Arbeitsintensivierung, Arbeitsverdichtung sowie höhere Anforderungen bezüglich der Entscheidungsgewalt und der arbeitsbezogenen Planung erleben (Kubicek et al. 2015). Der sog. ,Technostress“ (auch „Techopressure“ bzw. „Telepressure“) beschreibt eine Art technologisch-mediale Überforderung, aktuell meist untersucht anhand intensiver IKT-Nutzung und dem gleichzeitig empfundenen Druck, sofort auf z.B. eingehende Nachrichten reagieren zu müssen (Grawitch et al. 2018). Allerdings würde dieser Effekt auch durch interindividuelle Unterschiede, wie z.B. hohen Neurotizismus, niedrige Selbstkontrolle (Barber und Santuzzi 2017) und hohe Arbeitssucht erklärt und assoziiert mit der Angst, etwas Wichtiges zu verpassen, was wiederum mit Stress und Burnout zusammenhängt. Demgegenüber stehen Studien, die sich auch mit positiven Zusammenhängen auseinandersetzen: So könnte die Digitalisierung bei niedrig qualifizierten Beschäftigten sowie Beschäftig- 
ten mit körperlich hoch belastenden Tätigkeiten zu einer stärkeren körperlichen Entlastung führen als bei Höherqualifizierten sowie Beschäftigten ohne körperlich belastende Tätigkeiten (BMAS 2016). Führungskräfte (Gerten et al. 2018) und mobil arbeitende Beschäftigte (Tarafdar 2018), die hauptsächlich unterwegs arbeiten, würden von der IKTNutzung profitieren, da sie selbst bestimmen könnten, in welcher Weise sie die IKT nutzen und dabei persönliche Prioritäten setzen können. Fortgeschrittene digitale Fertigungstechnologien könnten zudem auch eine positive, persönlichkeitsförderliche Wirkung haben, wenn diese gleichzeitig z.B. mit intellektueller Stimulanz (Planung, Kontrolle und Problemlösung), erhöhtem Selbstmanagement, Engagement und Partizipation einhergehen (Bayo-Moriones et al. 2017). Generell ist die Studienlage über Stand und Wirkung digitaler Technologien jedoch v.a. jenseits von IKT noch recht übersichtlich. Die skizzierten unterschiedlichen Anforderungen implizieren zudem, dass auch der berufliche Kontext (z.B. die Branche oder die Tätigkeitsart) Unterschiede zwischen Beschäftigten in Hinblick auf die digitale Belastung erklären könnte. In einer Studie des BMWi wurden bei Vergleichen zwischen Finanzund Dienstleistungsbranchen, der Gesundheitsbranche und dem Fahrzeugbau grobe deskriptive Unterschiede im Grad der Digitalisierung beschrieben (BMWi 2018), jedoch keine weiteren inferenzstatistischen Unterscheidungen sowie Differenzierungen zwischen einzelnen Tätigkeitsfeldern innerhalb oder zwischen den Branchen betrachtet. Die vorliegende Studie möchte diese Lücke schließen und neben dem Stand der Digitalisierung (im Sinne einer Belastung) besagte Unterschiede in Hinblick auf ausgewählte Branchen und Tätigkeitsfelder untersuchen.

Auch Überwachung und Austauschbarkeit sowie beruflich-soziale Entkopplung sind Aspekte, die derzeit im Zuge der Digitalisierung kontrovers diskutiert werden. Formen der bewusst gesteuerten Überwachung sollen im Betrieb der Steigerung der Produktivität/Leistung, Sicherheit, sozialen Kontrolle sowie der Kreativität dienen (Martin und Freeman 2003), führen aber aufseiten der Beschäftigten häufig zu Unbehagen. Eine direkte Reaktion auf Überwachung (z.B. von E-Mails und Internetnutzung) ist häufig die Änderung des Verhaltens am Arbeitsplatz (Stanton und Weiss 2000), beispielsweise sinkt „unproduktives“ Verhalten (wie z.B. Internetnutzung für private Zwecke während der Arbeitszeiten). Allerdings wirkt digitale Überwachung auch negativ auf das subjektive Vertrauen der Beschäftigten zu ihrer Organisation und auf die wahrgenommene Gerechtigkeit der Überwachung (Alder et al. 2008). Hinzu kommen Befürchtungen über die Austauschbarkeit von Beschäftigten durch digitale Systeme. So hat sich der Anteil der Beschäftigten in Berufen mit einem hohen Substituierbarkeitspotenzial zwischen 2013 und 2016 bundesweit über alle Anforderungsniveaus und in fast allen Berufssegmenten erhöht (Dengler et al. 2018). Allerdings werden Veränderungen am Arbeitsmarkt eher i.S. von Verschiebungen von Arbeitsplätzen zwischen Berufen und Branchen diskutiert (Zika et al. 2018). Psychologisch interessant zu betrachten ist dabei, inwieweit sich berufliche Unsicherheit, Distanzierung und soziale Isolation zeigen - und inwieweit diese Einfluss auf die psychische Gesundheit nehmen. So ist aus der Untersuchung unbefriedigender und ungesicherter Beschäftigungsverhältnisse bekannt, dass sich diese genauso negativ wie Erwerblosigkeit auf die Gesundheit auswirken (Dooley 2003; Mohr und Duresso 2012).

Als wichtige zu berücksichtigende Variablen bei der Betrachtung von beruflichen Anforderungen und der persönlichen Gesundheit und Motivation (Van der Doef und Maes 1998) gelten allgemein arbeitsbezogene und persönliche Ressourcen wie z.B. die Arbeitsgestaltung, persönliche Autonomie und internale Kontrollüberzeugungen. Im Rahmen der Automatisierung zeigte sich, dass sich hoch automatisierte Systeme bei gleichzeitig geringem Einfluss (also dem Fehlen dieser Ressourcen) durchaus negativ auf die Beschäftigten auswirken (Jang et al. 2016). Auch die Perspektiven der Beschäftigten spielen eine wichtige Rolle: Mit dem Konzept der „digitalen Selbstwirksamkeit“ betrachten wir ein Konstrukt, das in Anlehnung an die allgemeine Selbstwirksamkeitserwartung (Bandura 1982, 1997) die Wahrnehmung der eigenen digitalen Fähigkeiten sowie die Überzeugung der Bewältigung digitaler Anforderungen umfasst (vgl. hierzu auch Moos und Azevedo 2009). Im Unterschied zu Studien, die umfassend und facettenreich über das Konzept der allgemeinen Selbstwirksamkeitserwartung als Ressource (Salanova et al. 2011; Shoji et al. 2016; Ventura et al. 2015) und Erfolgsfaktor (Carter et al. 2018; Maddux 2016) berichten, sind Studien zur Untersuchung von Selbstwirksamkeit im digitalisierten Arbeitskontext noch wenig verbreitet.

Schließlich ist im Kontext der Digitalisierung auch der Effekt auf das Wohlbefinden und die Work-Life-Balance der Beschäftigten von Interesse. Im Kontext der Digitalisierung könnten Tablets und andere IKT-Mobilgeräte dazu beitragen, dass mobiles Arbeiten und Remote-Work sich zunehmend verbreiten (Stawarz et al. 2013), was sich im Sinne von mehr zeitlicher und örtlicher Flexibilität von Beschäftigten einerseits durchaus positiv auf die Vereinbarkeit von beruflichen und privaten alltäglichen Anforderungen auswirken kann. Andererseits kann die häufige Nutzung von Smartphones und IKT für Arbeitszwecke in der Freizeit sowie die ständige Verfügbarkeit (Ninaus et al. 2015) und eine geringe Kontrolle über die Entgrenzung zwischen Arbeit und Privatleben zu einer hohen kognitiven Irritation (i. S. Nicht-Abschalten-Könnens) beitragen (Mellner 2016). Dies kann zu einer Einschränkung der Work-Life-Balance führen, die hier als mittel- bis langfristige Beanspruchungsfolge verstanden wird (vgl. z.B. Rusch 2019), sich auf ein 
ausgeglichenes Verhältnis der verschiedenen Lebenssphären in Berufs- und Privatleben bezieht (Hoff et al. 2005) und die gleichmäßige Involviertheit und Zufriedenheit mit dem Bewältigen der jeweils in Beruf- und Privatleben assoziierten Rollen beinhaltet (Schobert 2007). Eine eingeschränkte Work-Life-Balance kann somit als negative Konsequenz der Auswirkungen unterschiedlicher Stressoren und mangelnder Ressourcen betrachtet werden.

Es zeigt sich, dass bisher nur vereinzelte Studien zur Ausprägung und arbeitspsychologischen Zusammenhängen im Kontext der Digitalisierung existieren. Es stellt sich die Frage, was unter Digitalisierung der Arbeit in einzelnen Industriebranchen und Tätigkeitsfelder derzeit zu verstehen ist, welche Unterschiede derzeit existieren und welche arbeitspsychologischen Zusammenhänge erkennbar sind. Die vorliegende Studie verfolgt daher vor allem zunächst einen explorativen Weg und orientiert sich an folgenden Forschungsfragen:

1. Wie ist der aktuelle Stand der Digitalisierung in verschiedenen Industriebranchen in Deutschland und inwieweit werden bereits bestimmte digitale Technologien genutzt?

2. Wie wird die Digitalisierung von den Beschäftigten wahrgenommen? Gibt es hierbei Unterschiede zwischen den Industriebranchen und auch zwischen verschiedenen Tätigkeitsfeldern der Beschäftigten?

3. Welche psychologischen Zusammenhänge und Wirkmechanismen finden sich bei den Beschäftigten im Zuge der Digitalisierung?

Um diese Fragen zu beantworten, wurde eine deutschlandweite Studie durchgeführt, Beschäftigte aus unterschiedlichen Tätigkeitsfeldern befragt und für die Chemiebranche ein Strukturgleichungsmodell zur Analyse der komplexen Zusammenhänge erstellt. Gleichzeitig bildet die Studie den Ausgangspunkt für Längsschnittanalysen, die in den Folgejahren anschließen sollen.

\section{Methodisches Vorgehen und Datengrundlage}

Für die Analyse des aktuellen Status quo der Digitalisierung wurden deutschlandweit Beschäftigte aus zwölf Industriebranchen und acht verschiedenen Tätigkeitsfeldern untersucht (vgl. Tab. 1). Die Datenerhebung wurde als Online-Befragung durchgeführt. Landesweit wurden mit Unterstützung der „Industriegewerkschaft Bergbau, Chemie, Energie" (IG BCE) Beschäftigte in ihren Betrieben via Rundmail, Aushängen, Flyern, Infokarten und Betriebsversammlungen auf die Befragung hingewiesen. Dabei wurden ein URL-Link und QR-Code zur Website der Studie kommuniziert, auf der ein Link zum Start der Online-Befragung eingebettet war. Die Datenerhebung fand vom 01.02.2019 bis 31.03.2019 statt, die Beantwortung des Online-Fragebogens nahm durchschnittlich etwa $15 \mathrm{~min}$ in Anspruch. Sämtliche Daten wurden anonym erhoben und gespeichert, die Teilnahme an der Befragung war freiwillig, es wurden keine Incentivierungen vorgenommen.

Insgesamt klickten 16.346 Beschäftigte den Link zur Online-Befragung an. Nach der Datenbereinigung konnten für die Auswertung der Studie insgesamt 14.007 Befragte aus 614 Betrieben, zwölf Industriebranchen und allen Bundesländern berücksichtigt werden. Tab. 1 bietet einen Überblick über die Zusammensetzung des Samples. Ein Großteil der Befragten stammte aus westlichen und südlichen Bundesländern (dabei allein 51,9\% aus Nordrhein-Westfalen), aus nördlichen und östlichen Bundesländern nahmen vergleichsweise wenig Personen an der Befragung teil. Die Stichprobe war zudem geprägt durch einen großen Anteil von Befragten aus großen Betrieben ab 500 Mitarbeitenden (MA): Mehr als ein Drittel der Befragten (36\%) stammte aus Betrieben mit mehr als 5000 Beschäftigten. Aus kleinen und mittelständischen Betrieben mit unter 500 MA (KMU) stammten dagegen $23 \%$ der Befragten. Aus insgesamt 50 Einzelbetrieben nahmen mehr als 50 Personen an der Befragung teil, diese Betriebe stellten 76\% der Teilnehmenden. Das Alter der Befragten betrug im Durchschnitt 43,1 Jahre ( $\mathrm{SD}=12,0)$, der Frauenanteil lag insgesamt bei $34 \%$, in der Pharmabranche war er mit $42 \%$ am höchsten und im Zementbereich mit sechs Prozent am niedrigsten. Die acht voneinander abgegrenzten Tätigkeitsfelder verteilten sich breit: Verwaltung war mit $22 \%$ der Befragten anteilig am stärksten vertreten, gemeinsam mit Forschung \& Entwicklung, Leitung \& Planung sowie IT machten diese „White-Collar"-Bereiche $48 \%$ der Befragten aus. Personen aus den „Blue-Collar“-Tätigkeitsfeldern Produktion, Technik, Serviceleistung und Labor stellten zusammen $52 \%$ der Befragten. Das Gros der Befragten arbeitete unbefristet (92\%) und in Vollzeit (89\%). Je nach Branche lagen größtenteils voll- bzw. teilkontinuierliche Schichtarbeit vor, nur in den Branchen Kautschuk, Kunststoff und Keramik wurde mehrheitlich keine Schichtarbeit berichtet. Hervorzuheben ist das relativ hohe Qualifikationsniveau der Befragten: Lediglich $5 \%$ der Befragten gaben an, über keinen oder nur einen grundständigen Schulabschluss zu verfügen.

Der eigentliche Fragebogen bestand aus einer Vielzahl von Themen, arbeitspsychologischen Skalen sowie einigen ergänzenden Einzel-Items. Im ersten Teil wurde die Nutzung von 23 digitalen Technologien bei der eigenen Arbeit erfragt, untergliedert in vier Bereiche; das Antwortformat der angebotenen fünfstufigen Likertskala reichte von „nie“ bis ,sehr oft":

- Bereich 1 „Digitale Informations- und Kommunikationstechnologien": E-Mail, Intranet, internes soziales Netz- 
Tab. 1 Gesamtsample mit Rücklauf je Branche $\left(n_{1}\right)$, Bundesland $\left(n_{2}\right)$, Tätigkeitsfelder (\% im Sample)

Table 1 Total sample with response per sector $\left(n_{1}\right)$, federal state $\left(n_{2}\right)$, fields of work (\% in sample)

\begin{tabular}{|c|c|c|c|}
\hline Branchen & $n_{1}$ & $n_{2}$ & Tätigkeitsfelder \\
\hline $\begin{array}{l}\text { Chemie } \\
\text { Kunststoff } \\
\text { Pharmazie } \\
\text { Kautschuk } \\
\text { Glas } \\
\text { Keramik } \\
\text { Papier } \\
\text { Energie } \\
\text { Bergbau } \\
\text { Mineralöl } \\
\text { Zement } \\
\text { Sonstige } \\
\text { Ohne } \\
\text { Gesamt }\end{array}$ & $\begin{array}{r}9627 \\
216 \\
1515 \\
120 \\
107 \\
72 \\
527 \\
330 \\
144 \\
91 \\
37 \\
1064 \\
157 \\
\mathbf{1 4 . 0 0 7}\end{array}$ & & $\begin{array}{l}\text { Blue Collar } \\
\text { Produktion (16\%) } \\
\text { Technik }(16 \%) \\
\text { Service }(12 \%) \\
\text { Labor }(8 \%) \\
\text { White Collar } \\
\text { Verwaltung (22\%) } \\
\text { F \& E (14\%) } \\
\text { IT (7\%) } \\
\text { Leitung }(6 \%)\end{array}$ \\
\hline
\end{tabular}

werk/Messenger System, Smartphone/Tablet-Computer, Groupware, Videotelefonie und -konferenzen

- Bereich 2 „Digital aufbereitete Daten zu Endprodukten“: ERP-Systeme für Daten zu betrieblichen Ressourcen, CRM-Systeme für Kundendaten, Daten zu Produktlebenszyklen, digitale Verwaltungsprogramme zur Visualisierung und Berichtslegung, Big Data i. S. komplexer Datenmengen und -analysen, Künstliche Intelligenz i. S. selbstlernender ,intelligenter" Prozesse

- Bereich 3 „Digitale Technologien für Produktion, Instandhaltung und Arbeitsvorbereitung": Terminals mit Echtzeitdaten, mobile Arbeitsmittel mit Echtzeitdaten, 3D-Drucker, modulare Anlagen mit sensorengesteuerten ,intelligenten“ Robotern, Systeme mit Augmented bzw. Virtual Reality, Datenbrillen als optische Hilfsgeräte mit integrierten Display-Anzeigen

- Bereich 4 „Personenbezogene Messsysteme“: Gesundheits- bzw. Positions-Tracker, Smart Watch, Persönliche Schutzausrüstung (PSA) mit Messsensoren, Exoskelett

Anschließend wurden $\mathrm{zu}$ verschiedenen Themenbereichen der Studie entsprechende Skalen vorgelegt. Tab. 2 bietet einen Überblick über die Themenbereiche und Skalen sowie die jeweiligen Reliabilitäten und Iteminhalte als Antwortformat wurde stets eine fünfstufige Likertskala mit wechselnden verbalen Verankerungen angeboten. Da im Zuge der noch geringen Nutzung von Technologien der Bereiche 3 und 4 (vgl. deskriptive Befunde in Abb. 1) zu geringe Varianzen in den Daten vorlagen und die Voraussetzungen für faktorenanalytische Untersuchungen sowie anschließende Skalenbildungen dort nicht erfüllt waren, wurden lediglich die Items der Bereiche 1 und 2 für weiterführende Analysen verwendet, zu Skalen zusammengefasst und diese dementsprechend in die Skalenliste aufgenommen.

Bei der Datenauswertung wurden zunächst explorative Faktorenanalysen der Items mit schiefwinkliger Rotation durchgeführt, da für die hier gewählten und empirisch bereits belegten, aber ggfs. interkorrelierenden Dimensionen eine strenge Orthogonalitätsannahme nicht notwendig erschien. Die Faktorenanalysen beinhalteten Voraussetzungsprüfung der Stichprobeneignung (KMO-Index), den Bartlett-Test auf Sphärizität sowie Reliabilitätsprüfungen (Interkorrelationen der Items und Cronbach's Alpha), um die auszuwertenden Skalen zu verifizieren. Für die erstellten Skalen wurden anschließend Mittelwerte und Standardabweichungen berechnet sowie relative Häufigkeiten für die Nutzung der verschiedenen digitalen Technologien. Da sich im Zuge der Datenerhebung bei der soziodemografischen Analyse der Stichprobe gravierende Verzerrungseffekte hinsichtlich der Rückläufe in einzelnen Branchen im Vergleich zu den tatsächlichen Beschäftigtenzahlen des Statistischen Bundesamtes (2017) fanden, wurden die Daten aus unterund überrepräsentierten Gruppen so gewichtet, dass deren Verteilung innerhalb der Gesamtstichprobe der tatsächlichen prozentualen Verteilung in der Population entsprach. Somit konnte z. B. eine rücklaufbedingte überproportionale Dominanz der Chemiebranche in der Gesamtstichprobe und damit in den Ergebnissen der Studie vermieden werden. Für die Berechnungen innerhalb der Branchen und Tätigkeitsbereiche wurden aufgrund fehlender Vergleichswerte keine Gewichte verwendet.

Für die Untersuchung von Unterschieden zwischen den verschiedenen Branchen und zwischen den Tätigkeitsfeldern wurden für jede Skala Varianzanalysen und Effektstärkeuntersuchungen durchgeführt. Auch Unterschiede in Hinblick auf Betriebsgröße (von KMU unter 500 bis Be- 
Tab. 2 Themen der Studie mit Skalen, Reliabilitäten und Iteminhalten

Table 2 Themes of the study with scales, reliabilities and item contents

\begin{tabular}{|c|c|c|}
\hline Themen und Skalen & $r_{t t}$ & Iteminhalte \\
\hline \multicolumn{3}{|l|}{ Nutzung digitaler Arbeitsmittel ${ }^{\mathrm{a}}$} \\
\hline Digitale IKT & 0,76 & $\begin{array}{l}\text { E-Mail, Intranet, soziale Netzwerke/Messenger Systeme, Smartphone/Tablet-Computer, Groupware, } \\
\text { Videotelefonie }\end{array}$ \\
\hline $\begin{array}{l}\text { Digital aufbereitete Daten zu } \\
\text { Endprodukten }\end{array}$ & 0,79 & ERP, Produktlebenszyklus, Verwaltungsprogramme, CRM, Big Data, künstliche Intelligenz \\
\hline \multicolumn{3}{|l|}{ Digitale Arbeitsanforderungen ${ }^{\text {a }}$} \\
\hline $\begin{array}{l}\text { Anforderungszunahme durch } \\
\text { Digitale Systeme }\end{array}$ & 0,85 & $\begin{array}{l}\text { Herausforderungen an Kompetenzen, hohe zeitliche Flexibilität, schneller und mehr arbeiten, Gleich- } \\
\text { zeitigkeit mehrerer Aufgaben, anspruchsvollere Tätigkeiten }\end{array}$ \\
\hline $\begin{array}{l}\text { Überwachung und Aus- } \\
\text { tauschbarkeit }\end{array}$ & 0,85 & $\begin{array}{l}\text { Gefühl der Leistungskontrolle durch Einsatz digitaler Technologien, der Überwachung durch Daten- } \\
\text { sammeln, Austauschbarkeit, Abwertung als Fachkraft durch Einsatz digitaler Technologien }\end{array}$ \\
\hline Fremdbestimmung & 0,72 & $\begin{array}{l}\text { Digitale Technologien übernehmen Planung meiner Aufgaben, treffen für mich Entscheidungen, ich } \\
\text { brauche weniger Fähigkeiten }\end{array}$ \\
\hline \multicolumn{3}{|c|}{ Allgemeine Arbeitsanforderungen ${ }^{\mathrm{b}}$} \\
\hline Quantitative Belastung & 0,80 & Häufiger Zeitdruck, zu viel Arbeit, mehrere Aufgaben gleichzeitig zu bewältigen \\
\hline Qualitative Belastung & 0,64 & $\mathrm{Zu}$ schwierige Aufgaben, nicht genug ausgebildet \\
\hline $\begin{array}{l}\text { Zeitliche Flexibilitätsanforde- } \\
\text { rungen }\end{array}$ & 0,72 & $\begin{array}{l}\text { Stark schwankende tägliche Arbeitszeiten, nicht planbare Arbeitszeiten, notwendige Erreichbarkeit } \\
\text { in der Freizeit }\end{array}$ \\
\hline \multicolumn{3}{|l|}{ Beruflich-soziale Entkopplung ${ }^{\mathrm{c}}$} \\
\hline Berufliche Unsicherheit & 0,73 & $\begin{array}{l}\text { Sorgen vor Jobverlust, schwieriger Jobperspektive bei Arbeitslosigkeit sowie ungewollter Versetzung } \\
\text { auf andere Arbeitsstelle }\end{array}$ \\
\hline Berufliche Distanzierung & 0,86 & $\begin{array}{l}\text { Beruf ist fremd geworden, zunehmende Distanz und Gleichgültigkeit sowie fehlende Identifikation } \\
\text { mit der eigenen Tätigkeit }\end{array}$ \\
\hline Soziale Isolation & 0,69 & Persönlicher Austausch mit Kollegen fehlt, sozial nicht eingebunden, Einsamkeit \\
\hline \multicolumn{3}{|l|}{ Digitale Ressourcen } \\
\hline $\begin{array}{l}\text { Unterstützung \& Erleichte- } \\
\text { rung durch digitale Systeme }\end{array}$ & 0,79 & $\begin{array}{l}\text { Unterstützung bei Entscheidungen und Aufgabenplanung, Verfügbarkeit wichtiger Informationen, } \\
\text { Nutzung neuer Arbeitsformen, einfachere Vereinbarkeit von Berufs- und Privatleben durch digitale } \\
\text { Technologien }\end{array}$ \\
\hline Digitale Selbstwirksamkeit ${ }^{\mathrm{d}}$ & 0,70 & $\begin{array}{l}\text { Überzeugung zum Schritthalten mit der Digitalisierung, Zutrauen zur Bewältigung digitaler Anforde- } \\
\text { rungen, Umgang mit digitalen Technologien fällt leicht }\end{array}$ \\
\hline \multicolumn{3}{|l|}{ Allgemeine Ressourcen } \\
\hline Entscheidungsspielraum ${ }^{\mathrm{b}}$ & 0,91 & Selbstständig viele Entscheidungen treffen, Initiative und eigenes Ermessen \\
\hline Vollständigkeit ${ }^{\mathrm{b}}$ & 0,75 & Arbeitsvorgänge von Anfang bis Ende, klares Arbeitsergebnis am Aufgabenende \\
\hline Informationsaustausch ${ }^{\mathrm{b}}$ & 0,80 & Immer alle notwendigen Infos verfügbar, geregelte Informationsweitergabe \\
\hline Veränderungsbereitschaft ${ }^{\mathrm{e}}$ & 0,82 & $\begin{array}{l}\text { Offenheit gegenüber persönlichem Mehrwert sowie Freude über sich ergebende Veränderungen am } \\
\text { Arbeitsplatz }\end{array}$ \\
\hline \multicolumn{3}{|l|}{ Beanspruchungsfolgen } \\
\hline Kognitive Irritation $^{\mathrm{f}}$ & 0,92 & Zu Hause und im Urlaub an Schwierigkeiten bei der Arbeit denken, schwer abschalten \\
\hline Work-Life-Balance ${ }^{g}$ & 0,80 & $\begin{array}{l}\text { Zufriedenheit mit Balance zwischen Arbeit und Privatleben, gute Vereinbarkeit, Anforderungen glei- } \\
\text { chermaßen gut bewältigen können }\end{array}$ \\
\hline
\end{tabular}

aEigenkonstruktion der Skalen

bSkalen in Anlehnung an WDQ (Stegmann et al. 2010), SPA (Metz und Rothe 2017) sowie Härtwig und Sporbert (2013)

'Skalen in Anlehnung an Heinzer und Reichenbach (2013)

din Anlehnung an Jerusalem und Schwarzer (1986)

'In Anlehnung an Szebel (2015)

${ }^{\mathrm{f}}$ Mohr et al. (2007)

gIn Anlehnung an Syrek et al. (2011) sowie Härtwig und Sporbert (2013)

triebe mit über 5000 Beschäftigten), Geschlecht, Alter und Bildungsabschluss wurden betrachtet, allerdings fielen sie hier eher unsystematisch und gering aus, daher nahmen sie in dieser Studie keine zentrale Rolle ein. Um die komplexen Zusammenhänge der Skalen zu analysieren, wurde zudem ein Strukturgleichungsmodell spezifiziert. Für dessen
Entwicklung wurden die in der Faktorenanalyse extrahierten latenten Dimensionen in das Strukturgleichungsmodell aufgenommen, um Zusammenhänge der unabhängigen und abhängigen Dimensionen untereinander zu analysieren. Bei der Berechnung wurden ausschließlich Daten von Beschäftigten der Chemie-Branche verwendet, da es sich erstens um 


\section{Digitale IKT}

\begin{tabular}{|c|c|c|c|c|}
\hline E-Mail & $14 \because 77$ & ERP-Systeme & $47-12 \times 1312$ & $\begin{array}{l}\text { Terminals mit } \\
\text { Echtzeitdaten }\end{array}$ \\
\hline Intranet & 193434 & Produktzyklus & $4 \% 13 \times 159$ & $\begin{array}{l}\text { Remote } \\
\text { Control }\end{array}$ \\
\hline Social Media & $2117 \times 2023$ & $\begin{array}{l}\text { Verwaltungs- } \\
\text { programme }\end{array}$ & $46 \% 14 \quad 1410$ & 3D-Drucker \\
\hline $\begin{array}{r}\text { Smartphone / } \\
\text { Tablet }\end{array}$ & 36101529 & Big Data & $61813 \times 95$ & $\begin{array}{r}\text { Advanced } \\
\text { Robotic }\end{array}$ \\
\hline Groupware & $8314 \times 1715$ & CRM-Systeme & $62613 \times 87$ & Virtual Reality \\
\hline Videotelefon & $3618 \times 1614$ & $\begin{array}{l}\text { Künstliche } \\
\text { Intelligenz }\end{array}$ & 88 & Datenbrillen \\
\hline & $8 \mathrm{Ni}$ & Selten & $\otimes$ Gelegentlich & 因 Oft \\
\hline
\end{tabular}

\section{Digitale Technologien für die Produktion}

\section{Personenbezogene Messsysteme}
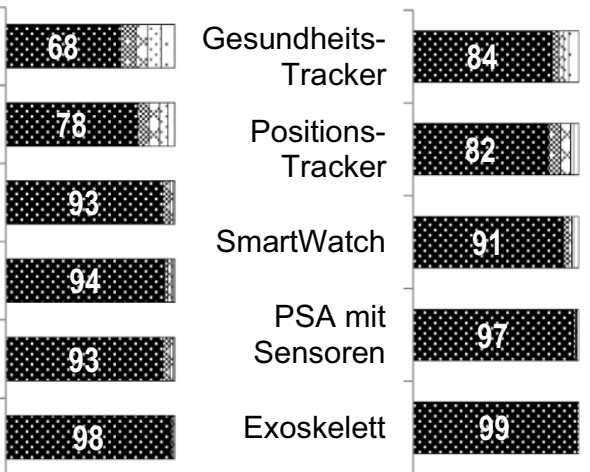

Abb. 1 Nutzung von Technologien der Digitalisierung (in Prozent der Beschäftigten)

Fig. 1 Usage of digitization technologies (in percent of employees)

die mit Abstand größte Teilgruppe im Sample handelte und um zweitens unsystematisch auftretende Verzerrungseffekte zu reduzieren, die die Entwicklung und Prüfung eines konvergierenden Modells verhindert hätten (vgl. Geiser 2011). Die Analysen wurden mit dem Programm MPlus 7.0 und in R 3.5.1 mit dem Paket lavaan durchgeführt. Alle Analysen wurden unter Verwendung des maximum-likelihoodSchätzers mit robusten Standardfehlern (MLR) umgesetzt. Für die Analysen im Rahmen der Strukturgleichungsmodellierung wurde der FIML (full information maximum likelihood)-Schätzer verwendet, der für die Schätzung der Modellparameter alle zur Verfügung stehenden Informationen heranzieht. Dieses Schätzverfahren ist bei zufälliger Verteilung fehlender Werte der paarweisen oder listenweisen Eliminierung bei der Modellparameterschätzung vorzuziehen (Schafer und Graham 2002).

\section{Ergebnisse}

\subsection{Status quo der Digitalisierung geprägt durch IKT}

Der erste Teil der Studie befasste sich mit dem Status quo der Digitalisierung im Sinne der tatsächlichen beruflichen Nutzung durch die Beschäftigten.

Abb. 1 fasst die Ergebnisse der Nutzungsabfrage zusammen. Die deskriptive Analyse zeigt, dass digitalisierte Arbeit derzeit v.a. die Nutzung von digitalen IKT bedeutet, da diese Technologien (Kat. 1) am häufigsten genutzt werden. V.a. E-Mail werden bei $91 \%$ der Beschäftigten ,,(sehr) oft" und Intranet bei $68 \%$,,(sehr) oft" genutzt und sind damit schon recht etabliert; bei den anderen IKT innerhalb dieser Kategorie zeigt sich das Nutzungsspektrum dage- gen als deutlich diverser. Auf entsprechende Unterschiede zwischen Branchen und Tätigkeitsfeldern wird im Folgeabschnitt eingegangen. Digital aufbereitete Daten zu Endprodukten (Kat. 2) werden in der Breite weniger häufig genutzt als IKT (z. B. ERP-Systeme von insg. $25 \%$,(sehr) oft"). Anwendungen für Big Data und Künstliche Intelligenz, die stark im Fokus der aktuellen medialen Diskussion stehen, werden von 61 bzw. $88 \%$,nie“ genutzt. Digitale Technologien für die Produktion (Kat. 3, z. B. 3D-Drucker und Virtual Reality mit je $93 \%$,nie“) und personenbezogene Messsysteme (Kat. 4, z.B. Gesundheits- oder PositionsTracker mit 84 bzw. $82 \%$ „nie“) finden bei nur wenigen Beschäftigten Anwendung.

\subsection{Positive Bewertungen und Tätigkeitsunterschiede in der digitalisierten Arbeit}

Der umfassendere zweite Teil der Studie befasste sich mit der Frage, inwieweit es Unterschiede zwischen den verschiedenen Industriebranchen und Tätigkeitsfeldern der Beschäftigten in Hinblick auf die Nutzung digitaler Technologien sowie Arbeitsanforderungen und Ressourcen gibt. Hierfür wurden die bereits oben betrachtete Nutzung digitaler Arbeitsmittel herangezogen, die digitalisierungsspezifischen und die allgemeinen Arbeitsanforderungen und Ressourcen, Aspekte der beruflich-sozialen Entkopplung sowie psychische Beanspruchungsfolgen und Wohlbefinden. Tab. 3 bietet einen Überblick über die Ergebnisse, dabei wird für jede Skala der Range der zwölf Branchen (mit Nennung der Branchen mit den beiden jeweils höchsten und niedrigsten Werten) sowie Unterschiedseffekte $\left(\eta^{2}{ }_{B}\right)$ berichtet, der Skalenmittelwert der gewichteten Gesamtstichprobe (Grand-Mean $\bar{X}$ ) sowie der Mittelwerte-Range der darin 
Tab. 3 Range der Skalenmittelwerte sowie Unterschiedseffekte auf Ebene der Industriebranchen und der Tätigkeitsfelder

Table 3 Range of scale means, difference effects on level of industrial sectors and on level of fields of work

\begin{tabular}{|c|c|c|c|c|c|}
\hline Themen und Skala & $\bar{X}(S D)$ & Industriebranchen (Range) & $\eta^{2} B$ & Tätigkeitsfelder (Range) & $\eta^{2} T$ \\
\hline \multicolumn{6}{|l|}{ Nutzung digitaler Arbeitsmittel } \\
\hline Digitale IKT & $\begin{array}{l}3,4 \\
(0,94)\end{array}$ & $\begin{array}{l}\text { Pharma, Papier ... Glas, Zement } \\
(3,8-2,1)\end{array}$ & 0,046 & $\begin{array}{l}\text { IT, Verwaltung ... Labor, Produktion } \\
(4,3-2,8)\end{array}$ & 0,212 \\
\hline Digital aufbereitete Daten & $\begin{array}{l}2,0 \\
(0,87)\end{array}$ & $\begin{array}{l}\text { Keramik, Sonstige ... Mineralöl, Zement } \\
(2,1-1,6)\end{array}$ & 0,009 & $\begin{array}{l}\text { IT, Leitung ... Labor, Technik } \\
(2,4-1,7)\end{array}$ & 0,039 \\
\hline \multicolumn{6}{|l|}{ Digitale Arbeitsanforderung } \\
\hline $\begin{array}{l}\text { Anforderungszunahme } \\
\text { durch digitale Systeme }\end{array}$ & $\begin{array}{l}2,7 \\
(0,90)\end{array}$ & $\begin{array}{l}\text { Papier, Pharma ... Zement, Mineralöl } \\
(2,8-2,2)\end{array}$ & 0,004 & $\begin{array}{l}\text { IT, Leitung ... Produktion, Labor } \\
(3,0-2,5)\end{array}$ & 0,028 \\
\hline $\begin{array}{l}\text { Überwachung \& Aus- } \\
\text { tauschbarkeit }\end{array}$ & $\begin{array}{l}2,5 \\
(1,0)\end{array}$ & $\begin{array}{l}\text { Energie, Glas ... Kautschuk, Zement } \\
(2,6-2,2)\end{array}$ & 0,003 & $\begin{array}{l}\text { Produktion, Service ... F \& E, Lei- } \\
\text { tung } \\
(2,6-2,2)\end{array}$ & 0,018 \\
\hline Fremdbestimmung & $\begin{array}{l}1,7 \\
(0,82)\end{array}$ & $\begin{array}{l}\text { Glas, Papier ... Zement, Mineralöl } \\
(1,9-1,5)\end{array}$ & 0,002 & $\begin{array}{l}\text { Service, Produktion ... Labor, F \& E } \\
(1,8-1,5)\end{array}$ & 0,011 \\
\hline \multicolumn{6}{|c|}{ Allgemeine Arbeitsanforderungen } \\
\hline Quantitative Belastung & $\begin{array}{l}3,5 \\
(0,80)\end{array}$ & $\begin{array}{l}\text { Kautschuk, Papier ... Zement, Bergbau } \\
(3,6-3,2)\end{array}$ & 0,005 & $\begin{array}{l}\text { Leitung, IT ... Produktion, Labor } \\
(3,7-3,3)\end{array}$ & 0,014 \\
\hline Qualitative Belastung & $\begin{array}{l}2,0 \\
(0,85)\end{array}$ & $\begin{array}{l}\text { Zement, Glas ... Sonstige, Bergbau } \\
(2,2-1,9)\end{array}$ & 0,002 & $\begin{array}{l}\text { Technik, IT ... Leitung, Labor } \\
(2,1-1,9)\end{array}$ & 0,010 \\
\hline $\begin{array}{l}\text { Zeitliche Flexibilitätsanfor- } \\
\text { derungen }\end{array}$ & $\begin{array}{l}2,2 \\
(0,92)\end{array}$ & $\begin{array}{l}\text { Papier, Mineralöl ... Keramik, Bergbau } \\
(2,4-2,0)\end{array}$ & 0,006 & $\begin{array}{l}\text { Leitung, IT ... Labor, Produktion } \\
(2,6-2,0)\end{array}$ & 0,035 \\
\hline \multicolumn{6}{|l|}{ Beruflich-soziale Entkopplung } \\
\hline Berufliche Unsicherheit & $\begin{array}{l}2,3 \\
(0,97)\end{array}$ & $\begin{array}{l}\text { Pharma, Zement ... Kautschuk, Mineralöl } \\
(2,4-1,8)\end{array}$ & 0,004 & $\begin{array}{l}\text { IT, Service ... Technik, Leitung } \\
(2,4-2,1)\end{array}$ & 0,011 \\
\hline Berufliche Distanzierung & $\begin{array}{l}1,7 \\
(0,76)\end{array}$ & $\begin{array}{l}\text { Pharma, Chemie ... Bergbau, Kautschuk } \\
(1,8-1,6)\end{array}$ & 0,004 & $\begin{array}{l}\text { Produktion, IT ... F \& E, Leitung } \\
(1,8-1,6)\end{array}$ & 0,004 \\
\hline Soziale Isolation & $\begin{array}{l}1,9 \\
(0,74)\end{array}$ & $\begin{array}{l}\text { Zement, Mineralöl ... Keramik, Kau- } \\
\text { tschuk } \\
(2,2-1,8)\end{array}$ & 0,001 & $\begin{array}{l}\text { Produktion, Service ... Verwaltung } \\
\text { F\&E } \\
(2,0-1,8)\end{array}$ & 0,007 \\
\hline \multicolumn{6}{|l|}{ Digitale Ressourcen } \\
\hline $\begin{array}{l}\text { Unterstützung \& Erleichte- } \\
\text { rung }\end{array}$ & $2,8(0,96)$ & $\begin{array}{l}\text { Kautschuk, Pharma ... Glas, Zement } \\
(3,2-2,2)\end{array}$ & 0,019 & $\begin{array}{l}\text { IT, Leitung ... Labor, Produktion } \\
(3,6-2,4)\end{array}$ & 0,163 \\
\hline Digitale Selbstwirksamkeit & $\begin{array}{l}3,9 \\
(0,79)\end{array}$ & $\begin{array}{l}\text { Kautschuk, Sonstige ... Energie, Zement } \\
(4,0-3,7)\end{array}$ & 0,005 & $\begin{array}{l}\text { IT, Leitung ... Produktion, Service } \\
(4,2-3,8)\end{array}$ & 0,018 \\
\hline \multicolumn{6}{|l|}{ Allgemeine Arbeitsressourcen } \\
\hline Entscheidungsspielraum & $\begin{array}{l}3,5 \\
(0,96)\end{array}$ & $\begin{array}{l}\text { Kautschuk, Papier ... Pharma, Bergbau } \\
(3,9-3,4)\end{array}$ & 0,005 & $\begin{array}{l}\text { Leitung, IT ... Labor, Produktion } \\
(3,9-3,1)\end{array}$ & 0,049 \\
\hline Vollständigkeit & $\begin{array}{l}3,9 \\
(0,90)\end{array}$ & $\begin{array}{l}\text { Zement, Papier ... Kautschuk, Mineralöl } \\
(4,1-3,7)\end{array}$ & 0,001 & $\begin{array}{l}\text { Labor, Technik ... Produktion, IT } \\
(4,1-3,7)\end{array}$ & 0,022 \\
\hline Informationsaustausch & $\begin{array}{l}2,9 \\
(0,82)\end{array}$ & $\begin{array}{l}\text { Chemie, Sonstige ... Glas, Kunststoff } \\
(3,0-2,8)\end{array}$ & 0,003 & $\begin{array}{l}\text { Labor, F \& E ... IT, Technik } \\
(3,1-2,8)\end{array}$ & 0,011 \\
\hline Veränderungsbereitschaft & $\begin{array}{l}3,6 \\
(0,80)\end{array}$ & $\begin{array}{l}\text { Kautschuk, Glas ... Zement, Energie } \\
(3,8-3,3)\end{array}$ & 0,006 & $\begin{array}{l}\text { Leitung, IT ... Produktion, Technik } \\
(3,7-3,4)\end{array}$ & 0,012 \\
\hline \multicolumn{6}{|l|}{ Beanspruchungsfolgen } \\
\hline Kognitive Irritation & $\begin{array}{l}2,5 \\
(1,1)\end{array}$ & $\begin{array}{l}\text { Papier, Zement ... Glas, Bergbau } \\
(2,7-2,3)\end{array}$ & 0,002 & $\begin{array}{l}\text { Leitung, IT ... Produktion, Labor } \\
(2,7-2,2)\end{array}$ & 0,011 \\
\hline Work-Life-Balance & $\begin{array}{l}3,6 \\
(0,84)\end{array}$ & $\begin{array}{l}\text { Bergbau, Keramik ... Pharma, Mineralöl } \\
(3,7-3,3)\end{array}$ & 0,002 & $\begin{array}{l}\text { F \& E, Labor ... Service Produktion } \\
(3,7-3,5)\end{array}$ & 0,008 \\
\hline
\end{tabular}

Zur Berechnung des Grand-Means $(\bar{X})$ wurden die Daten anhand der tatsächlichen Beschäftigtenanzahlen gewichtet. Das Antwortformat entsprach stets einer fünfstufigen Likertskala von 1 bis 5 mit wechselnden verbalen Verankerungen. Die Skalen sind so gepolt, dass hohe Werte für hohe Ausprägungen stehen. Markierter Wertebereich für Unterschiedseffekt $\eta^{2}$ : kein Effekt $<0,010$, kleiner Effekt $\geq 0,010$, mittlerer Effekt $\geq \mathbf{0 , 0 6 0}$, großer Effekt $\geq 0,140$ 
voneinander differenzierten acht Tätigkeitsfelder mitsamt den dazugehörigen Unterschiedseffekten $\left(\eta^{2} T\right)$. Die Ergebnisse zeigen mehrerlei:

Erstens wurde die Digitalisierung und die damit assoziierten Herausforderungen von den Befragten insgesamt durchaus positiv eingeschätzt und weniger als Bedrohung gesehen, als es in Anbetracht der Diskussion möglicher Entwicklungsszenarien zu vermuten gewesen wäre (vgl. Hirsch-Kreinsen 2016). Dies zeigen zum einen die insgesamt eher niedrigen Ausprägungen der digitalisierungsspezifischen negativ assoziierten Konstrukte „Fremdbestimmung“ (Grand Mean $\bar{X}=1,7)$, „Überwachung“ (2,5) und die eher hoch ausgeprägte Ressource „Digitale Selbstwirksamkeit“ $(3,9)$, als auch die niedrig ausgeprägte allgemeine Berufliche Unsicherheit (2,3), Berufliche Distanzierung $(1,7)$ und Soziale Isolation $(1,9)$. Eher ambivalent bzw. mäßig bewertet wurden die Unterstützung und Erleichterung $(2,8)$ sowie die Anforderungszunahme durch digitale Systeme $(2,7)$.

Zweitens zeigt sich, dass Unterschiede zwischen den hier untersuchten Industriebranchen in Anbetracht niedriger Effektstärken $\eta^{2}{ }_{B}$ eher zu vernachlässigen sind. Dagegen fallen auf Ebene der verschiedenen Tätigkeitsfelder Unterschiedseffekte $\eta^{2}{ }_{T}$ stärker ins Gewicht. Bei den Branchen zeigten sich lediglich bei der ,Nutzung digitaler IKT-Systeme“ $\left(\eta^{2}{ }_{B}=0,046\right)$ und der ,Unterstützung und Erleichterung durch digitale Systeme“ $(0,019)$ gering bedeutsame Unterschiedseffekte, denen zufolge in den Industriebbereichen Kautschuk, Papier und Pharma jeweils höhere Werte erzielt wurden als bei Zement, Glas und Bergbau - die anderen Branchen verteilten sich zwischen diesen Extrema um den jeweiligen Grand-Mean. Deutlich prägnanter fielen dagegen Unterschiedseffekte zwischen den einzelnen Tätigkeitsfeldern aus, vor allem bei der ,Nutzung digitaler IKT“ $\left(\eta^{2}{ }_{T}=0,212\right)$, der ,Nutzung digital aufbereiteter Daten“ (0,039), der ,Unterstützung und Erleichterung“ $(0,163)$ sowie der ,Anforderungszunahme durch digitale Systeme“ $(0,028)$. So offenbarte sich wiederholt eine gewisse Dualität zwischen sog. White-Collar-Tätigkeitsfeldern (IT, Leitung \& Planung, Verwaltung, Forschung \& Entwicklung) und Blue-Collar-Tätigkeitsfeldern (Produktion, Technik, Labor, Serviceleistung): Beschäftigte mit vornehmlich planerischadministrativen Büro-Tätigkeiten waren häufiger bzw. stärker mit den Nutzungsherausforderungen und Anforderungszunahmen der Digitalisierung konfrontiert als Beschäftigte in handwerklich-technischen Tätigkeiten, zeigten aber auch etwas günstigere Werte bei der empfundenen „Überwachungen \& Austauschbarkeit" und den digitalisierungsspezifischen Ressourcen. Demgegenüber ließen sich nur sehr geringe Unterschiedseffekte in den Skalen der Beanspruchungsfolgen und der beruflich-sozialen Entkopplung erkennen - sowohl auf Ebene der Branchen, als auch auf Ebene der Tätigkeitsfelder.
Zur Einordnung der Ergebnisse (und v.a. für Zusammenhangsuntersuchungen im unten folgende Strukturgleichungsmodell) wurden auch allgemeine und eher digitalisierungsunspezifische Belastungs- und Gestaltungsfaktoren der Arbeit untersucht. Hier zeigten sich sowohl leicht erhöhte Werte in der „quantitativen Belastung“ $(\bar{X}=3,5)$, als auch eher geringe und damit positive Werte in der ,qualitativen Belastung“ $(2,0)$ und in ,zeitlichen Flexibilitätsanforderungen“ (2,2). Auch die klassischen Gestaltungsfaktoren ,,Vollständigkeit“ $(3,9)$ und „Entscheidungsspielraum“ $(3,5)$ waren eher positiv ausgeprägt, „Informationsaustausch“ (2,9) dagegen mäßig. In allen genannten Bereichen zeigten sich gering bedeutsame Unterschiedseffekte zwischen den verschiedenen Tätigkeitsfeldern mit etwas höheren Werten in den White-Collar-Tätigkeiten, Branchenunterschiede waren demgegenüber nicht bedeutsam.

\subsection{Zusammenspiel von Belastung, Ressourcen, Beruflich-sozialer Entkopplung und Gesundheit in der Digitalisierung}

Der dritte Teil der Studie beinhaltete die Entwicklung eines Strukturgleichungsmodells (vgl. Abb. 2), um empirische Zusammenhänge der digitalisierungsspezifischen und allgemeinen Themenbereiche in der Chemiebranche darzustellen und gleichzeitig Gestaltungsimpulse für den betrieblichen Kontext abzuleiten. Das Modell weist in der Gesamtschau der Kennwerte einen akzeptablen bis guten Modellfit auf $\left(C F I=0,92, R M S E A=0,04, S R M R=0,07, x^{2}=15.421 .010\right.$, $d f=973, p<0,001)$, die abgebildeten standardisierten Faktorladungen waren für alle eingeschlossenen Dimensionen statistisch signifikant und substanziell, Dimensionen ohne substanziellen Beitrag wurden nicht in das Modell integriert und daher nicht weiter aufgeführt.

Das entwickelte Modell passt sich in den theoretischen Rahmen der Studie des Job-Demands-Resources-Modell (Bakker und Demerouti 2007, 2014) ein und ergänzt es sowohl um digitalisierungsspezifische Aspekte, als auch um den als Stressor interpretierbaren Bereich der Beruflich-sozialen Entkopplung. Im oberen und mittleren Teil des Modells (vgl. Abb. 2) zeigen sich Wirkzusammenhänge, die vergleichbar sind zu jenen des JDR-Modells: Die digitalisierungsspezifischen und allgemeinen beruflichen Anforderungen zeigen substanzielle Interkorrelationen und wirken theoriekonform mit jeweils positiven standardisierten $\beta$-Regressionskoeffizienten $(0,09,0,41,0,22)$ auf die negativ konnotierte Beanspruchungsfolge „Kognitive Irritation" sowie mit negativen $\beta(-0,40$ und $-0,11)$ auf die positive konnotierte Beanspruchungsfolge „,Work-LifeBalance“. Gleichzeitig leisten die persönlichen Ressourcen (hier „Digitale Selbstwirksamkeit“) mit negativem $\beta=-0,10$ einen Beitrag zur Erklärung der „Kognitiven Irritation“ und die berufliche Ressource „Informationsaustausch“ mit 
Abb. 2 Strukturgleichungsmodell für die Chemie-Branche

Fig. 2 Structural equation model for the chemical industry

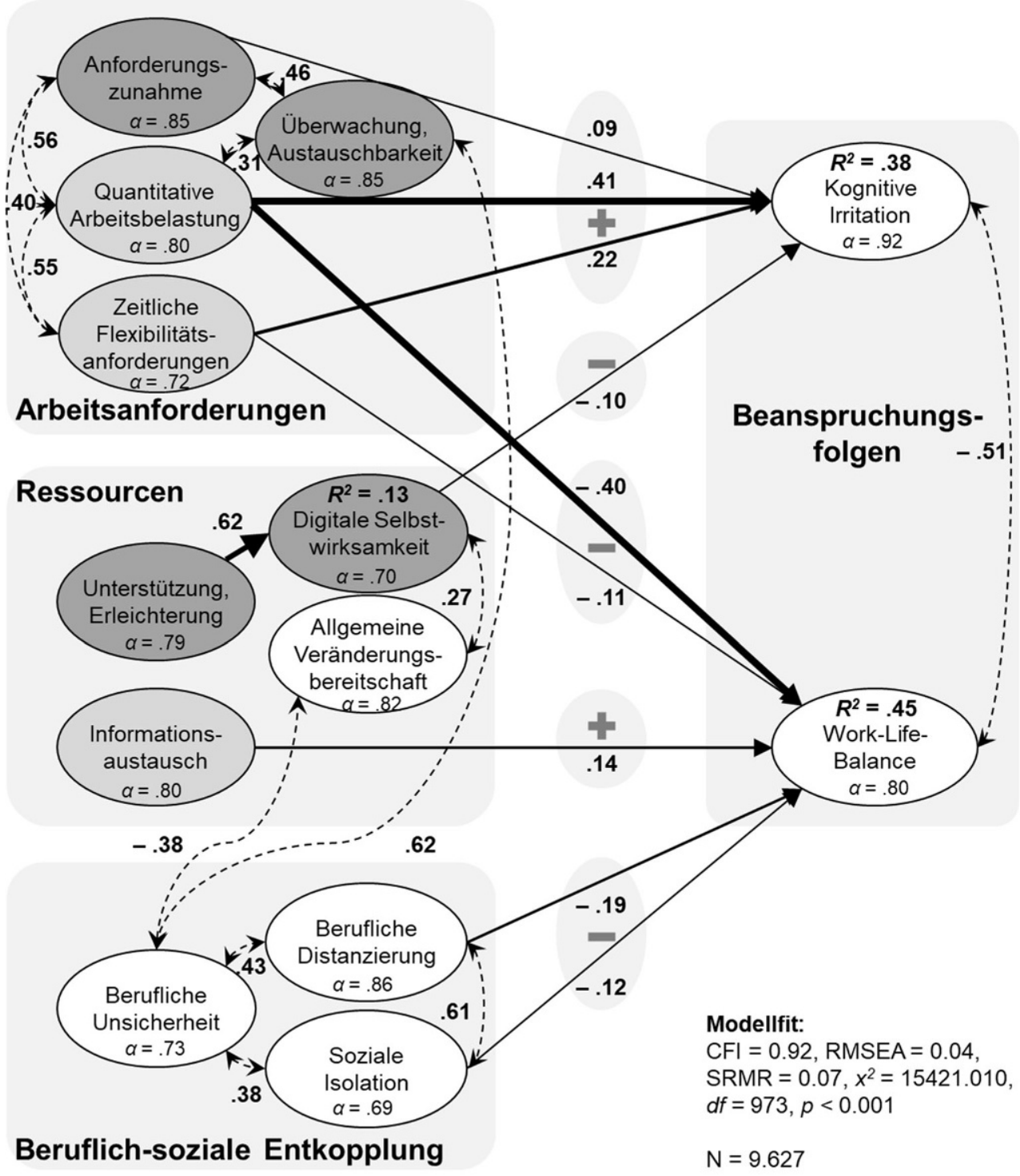

Digitalisierung positivem $\beta=0,14$ einen Beitrag zur Erklärung der Beanspruchungsfolge „Work-Life-Balance“. Zudem zeigt sich, dass die Ressource „Digitale Selbstwirksamkeit“ selbst wieder durch die betriebliche „Unterstützung und Erleichterung durch digitale Systeme" mit $\beta=0,62$ erklärt wird $\left(R^{2}=0,13\right)$ - dies kann auch als Mediationseffekt interpretiert werden, der die Unterstützung digitaler Systeme bei der Reduzierung kognitiver Irritation über die Digitale Selbstwirksamkeit erklärt. Ergänzt wird das Modell nun um die als Stressoren interpretierbaren Aspekte der beruflich-sozialen Entkopplung, die sich aus den substanziell miteinander interkorrelierenden Variablen „Berufliche Unsicherheit", „Berufliche Distanzierung“ und „Soziale Isolation" zusammensetzen; letztere wirken mit negativem $\beta$ $(-0,19$ und $-0,12)$ auf „Work-Life-Balance“. Insgesamt werden in diesem Modell 38\% der Varianz der Variable „Kognitive Irritation“ erklärt und $45 \%$ der Variable „WorkLife-Balance“. Die „Berufliche Unsicherheit“ stellt sich in dieser Studie zudem als eine Art Verbindungsglied dar: Sie korreliert einerseits zu $r=0,62$ stark positiv mit der digitalisierungsrelevanten „Überwachung und Austauschbarkeit" und andererseits zu $r=-0,38$ mit der persönlichen Ressource „Allgemeine Veränderungsbereitschaft“. Somit zeigt sich, dass die Aspekte der sozialen Einbindung und beruflichen Identität (wie bereits ganz allgemein im beruflichen Erleben) auch im Kontext der Digitalisierung einen zu berücksichtigenden Beitrag zum Verständnis von psychischen Beanspruchungsfolgen leisten. 


\section{Diskussion des methodischen Vorgehens und der Ergebnisse}

Die Digitalisierung ist Teil unseres Alltags und verändert Arbeitsprozesse und die Arbeitswelt, sodass sich Betriebe und Beschäftigte mit den damit einhergehenden Chancen und Herausforderungen auseinandersetzen müssen (Clauß und Verworn 2019). Die vorliegende Studie hatte zum Ziel, den Status quo sowie Anforderungen und Auswirkungen der Digitalisierung in zwölf Industriebranchen aus Sicht der Beschäftigten zu untersuchen, Unterschiede zwischen den Branchen sowie zwischen acht verschiedenen Tätigkeitsfeldern zu analysieren und empirische Zusammenhänge und Wirkeinflüsse der erfassten Aspekte im Rahmen aktueller theoretischer Modelle zu ergründen.

Bei der Betrachtung der Ergebnisse sind zunächst einige Limitationen zu berücksichtigen: Zwar verhelfen die thematische Fülle, die große Anzahl an Teilnehmenden und der differenzierte Querschnitt über zwölf Industriebranchen und acht Tätigkeitsfelder sowie die Verknüpfung der Daten mithilfe des Strukturgleichungsmodells zu einer vielversprechenden Datengrundlage, um fundierte Aussagen für den anvisierten Geltungsbereich in den untersuchten Branchen und Tätigkeitsfeldern zu treffen. Trotz der guten Datengrundlage sollten bei der Ergebnisinterpretation berücksichtigt werden, dass Verzerrungseffekte durch das gewählte Erhebungsmedium der Online-Befragung denkbar sind: Zugang und Affinität zu digitalen Technologien (immerhin Hauptgegenstand der Studie) sowie deren Bedienung könnten die Wahrscheinlichkeit zur Teilnahme an der Befragung durchaus beeinflusst haben - eine parallele Befragung z. B. anhand klassischer Paper-Pencil-Fragebögen wäre also durchaus hilfreich gewesen. Auch sind Stichprobeneffekte denkbar: Zwar konnten Verzerrungen auf Ebene der Branchen durch die beschriebenen Gewichtungen kompensiert werden, auf Ebene der Tätigkeitsfelder war dies wegen fehlender Vergleichsdaten jedoch nicht möglich. Zu diskutieren ist auch, dass das Sample durch einen hohen Anteil von qualifizierten Beschäftigten aus (für die Prozessindustrie durchaus typischen) großen Betrieben im Süden und Westen des Landes sowie Personen mit unbefristeten Beschäftigungsverhältnissen geprägt ist. Daher wären differenziertere Untersuchungen von Beschäftigten in KMU lohnend, ebenso von Personen mit fragileren Beschäftigungskonstellationen sowie Personen mit niedrigerem Bildungshintergrund. Auch ein Effekt durch die studienunterstützende Organisation im Sinne induzierter sozial erwünschter Antworten der Befragten ist prinzipiell denkbar, ansonsten können auch hier die üblichen Einschränkungen von Selbstberichtsdaten diskutiert werden. Für das Strukturgleichungsmodell wurden aus methodischen Gründen ausschließlich Beschäftigte der Chemiebranche herangezogen, um die Stabilität des Modells sicherzustellen, was den Transfer der Befunde zunächst etwas eingrenzen könnte. Jedoch konnten keine systematischen Einflüsse der Branchenart identifiziert werden - zudem zeigten sich in der Studie insgesamt nur geringe bis keine Branchenunterschiede, sodass eine Anwendung des Modells auch in anderen Bereichen diskutierbar ist.

Trotz dieser zu berücksichtigenden Einschränkungen wird deutlich, dass die Digitalisierung in Hinblick auf den Status quo bei der Breite der Beschäftigten noch am Anfang steht und sich eher auf die Nutzung digitaler IKT sowie digital aufbereiteter Daten eingrenzen lässt. Dies bestätigt Daten des Statistischen Bundesamtes, nach denen in Deutschland z. B. die E-Mail-Nutzung von 2000 bis 2018 exponentiell gestiegen ist (Statistisches Bundesamt 2019). Dagegen werden jene digitalen Technologien, die häufig im Fokus der medialen Berichterstattung liegen und als typische Beispiele der Digitalisierung in der Arbeitswelt angeführt werden wie z.B. Big Data, Künstliche Intelligenz, 3D-Drucker, Virtual Reality, Tracking-Systeme und Exoskelette bisher tatsächlich eher noch sporadisch genutzt. Bereits in einer Studie des BMWi wurden bei globaleren Vergleichen z.B. zwischen wissensintensiven Finanz- und Dienstleistungsbranchen, der Gesundheitsbranche und dem Fahrzeugbau deskriptive Unterschiede im Grad der Digitalisierung beschrieben (BMWi 2018), inferenzstatistische Unterscheidungen sowie Differenzierungen zwischen einzelnen Tätigkeitsfeldern innerhalb der Branchen standen dagegen weniger im Fokus. Auch zeigte sich eine gewisse Vermischung der Dimensionen Branche und Tätigkeit. Die nun hier durchgeführte Studie zeigt dagegen, dass Unterschiede zwischen den hier untersuchten Industriebranchen deutlich geringer ausfallen, als dies in Ansehung entsprechender Publikationen zu vermuten gewesen wäre. Dies erscheint durchaus plausibel, da sich Tätigkeiten vor allem an expliziten Berufen orientieren, die nach Lempert (2006) als Inbegriff spezialisierter Anforderungen und Tätigkeiten $\mathrm{zu}$ verstehen sind, deren Ausübung die Ausbildung besonderer sensumotorischer und intellektueller Fähigkeiten voraussetzt und zudem persönlichkeitsbildende Sozialisationseffekte sowie eine erfahrungsbedingte Erweiterung des Wissens, Könnens und der Handlungsfähigkeit in Ansehung der jeweiligen Tätigkeit und Umwelt beinhalten. Infolgedessen ähneln sich Angestellte spezifischer Berufe (z.B. in der Technik, Verwaltung, IT) auch über Branchengrenzen hinweg eher als z.B. Angestellte verschiedener Berufe innerhalb einer Branche (z.B. Chemie, Energie, Pharmazie). In unserer Studie war bei Beschäftigten in sog. White-Collar-Tätigkeitsfeldern die Digitalisierung bereits präsenter als in Blue-Collar-Berufen, was sowohl die Nutzung der digitalen Arbeitsmittel und Anforderungszunahme betrifft, als auch die wahrgenommene Unterstützung durch digitale Systeme - berufspraktisch gehen diese Aspekte eng miteinander einher. Daher sollten Besonderheiten und Unterschiede der Tätigkeitsfelder auch bei der Diskussion 
der Anforderungen und Konsequenzen der Digitalisierung stärker berücksichtigt werden.

Bemerkenswerterweise zeigen sich in der Breite der hier Befragten insgesamt eher niedrige Befürchtungen und eine durchaus positiv ausgeprägte Zuversicht in Hinblick auf die digitalen Veränderungen, was aus psychologischer Perspektive als wichtige Arbeitsressource zur Bewältigung des Wandels zu verstehen ist. Vor allem Selbstwirksamkeit ist eine wichtige Ressource, die angibt, wie stark Beschäftigte überzeugt sind, bestimmte Arbeitsaufgaben zu meistern (Spreitzer 1995). Digitale Selbstwirksamkeit ist hier nun als eine Anwendung des klassischen Konstruktes von Selbstwirksamkeit (Bandura 1982, 1997) auf den Kontext der Digitalisierung zu verstehen. Basierend auf den inferenzstatistischen Ergebnissen des Strukturgleichungsmodells konnte gezeigt werden, dass digitale Selbstwirksamkeit sowohl mit allgemeiner Veränderungsbereitschaft signifikant positiv korreliert, als auch als Ressource sowohl mildernden Einfluss auf die Kognitive Irritation nimmt und mediierend die unterstützende Funktion digitaler Systeme erklärt. Solche Systeme entfalten demnach vor allem dann ihre unterstützende Wirkung, wenn sie bei den Nutzenden auch das Zutrauen stärken, die gestellten digitalen Anforderungen zu bewältigen, wenn ihnen der Umgang mit der jeweiligen Technologie leichtfällt und sie die Überzeugung haben, mit der Digitalisierung Schritt zu halten. Die vorgelegte Studie fügt sich daher in Arbeiten ein, die andere Selbstwirksamkeitsdimensionen näher untersuchten: So konnte z. B. ein Zusammenhang zwischen selbst-orientierter Selbstwirksamkeit und emotionaler Irritation gefunden werden (Loeb et al. 2016); zudem zeigten Sonnentag und Kruel (2006), dass erholungsbezogene Selbstwirksamkeit die Fähigkeit des Abschaltens beeinflusst. Unsere Ergebnisse konnten daher die bestendenden Befunden zur Bewältigung digitalisierungsspezifischer Anforderungen erweitern.

Aus theoretischer Perspektive kann die vorliegende Studie insofern einen Beitrag leisten, als dass auftretende Belastungs-Beanspruchungs-Konstellationen auch im Kontext der Digitalisierung empirisch und theoretisch erklärt werden können. Ergänzend zum klassischen Belastungs-Beanspruchungsmodell (Hackman und Oldham 1980) sowie JDR-Modell (vgl. im Überblick Demerouti und Nachreiner 2019) konnte gezeigt werden, dass hier sowohl digitalisierungsspezifische, als auch allgemeine Arbeitsanforderungen und Ressourcen bei der Erklärung von psychischen Beanspruchungsfolgen berücksichtigt werden müssen. Dabei wurde die digitale Selbstwirksamkeit als Ressource identifiziert, die zudem als Mediator die Wirksamkeit unterstützender digitaler Systeme erklärt. Zudem wurden in diesem Kontext auch Aspekte der beruflich-sozialen Entkopplung mit drohender Verunsicherung und Erosion von Bindung an Tätigkeit, Beruf und berufliche Gemeinschaft als beeinflussende Phänomene von Work-Life-Balance identifiziert.
Dies ist insofern relevant, als dass aus der CommitmentForschung bekannt ist, dass die berufliche Bindung eine durchaus wichtige psychologische und auch identitätsstiftende Ressource im Beruf darstellt (vgl. z. B. Berg 2017). Auch soziale Zugehörigkeit im beruflichen Kontext ist ein wichtiges Grundbedürfnis und dient als bedeutsame organisationale Ressource (Becke 2018). Dagegen ist Ausgrenzung im Arbeitskontext mit zahlreichen negativen Folgen für Beschäftigte und die Organisation assoziiert: Betroffene Mitarbeitenden leiden unter dem verminderten Selbstwertund Autonomiegefühl, fühlen sich nicht zugehörig, haben oft existenzielle Krisen und sind auch weniger produktiv (Ferris et al. 2008). So wirken sich ungesicherte Beschäftigungsverhältnisse vergleichbar negativ wie Erwerblosigkeit auf die psychische Gesundheit aus (Dooley 2003; Mohr und Duresso 2012). Dies zeigt, dass erodierende berufliche Bindung und die identitätsstiftende Funktion von Beruf auch im Kontext der Digitalisierung mitberücksichtigt werden sollte. Im Zuge sich weiter ändernder Berufsbilder, Tätigkeitsspektren, Veränderung von Teamstrukturen und betrieblicher Prozesse sollten Zusammenhänge mit beruflicher Unsicherheit, beruflicher Distanzierung und sozialer Isolation also weiter untersucht werden. Um diese und weitere etwaige Veränderungseffekte im Zuge der voranschreitenden Digitalisierung präzise zu untersuchen, wären auch Längsschnittanalysen durchaus lohnend. So könnten auch Adaptations- und Assimilationsprozesse, Effekte im Zuge des demografischen Wandels, Untersuchungen im Zuge der angeschnittenen Dualität von Blue- und White-Collar-Tätigkeiten sowie Veränderungseffekte im Zuge der Weiterentwicklung von Berufen sowie des Systems der Berufe untersucht werden.

\section{Praktische Implikationen}

In der vorliegenden Studie weisen wir auf gefundene Unterschiede v.a. in der Nutzung digitaler IKT und in der empfundenen Unterstützung und Erleichterung durch digitale Technologien zwischen Blue- und White-Collar-Beschäftigten hin. Um Beschäftigte aller Bereiche angemessen zu unterstützen und möglichen Polarisierungen entgegenzuwirken (Windelband 2019; Windelband und Spöttl 2018), sollten passende betriebliche Maßnahmen für die jeweiligen Gruppen entwickelt werden, die die spezifischen Anforderungen und Bedürfnisse berücksichtigen. Basierend auf den Ergebnissen des Strukturgleichungsmodells sollten bei der Regulierung von Belastungen im Zuge der Digitalisierung sowohl digitalisierungsspezifische, als auch allgemeine Arbeitsanforderungen sowie die Förderung betrieblicher und persönlicher digitaler Ressourcen im Fokus stehen. Zu empfehlen ist, neben der Implementierung hilfreicher digitaler Systeme gleichzeitig auch ein Fokus auf die (hier als 
wichtiger Mediator identifizierte) digitale Selbstwirksamkeit. Die Weiterentwicklung digitaler Kompetenzen ist hierbei ein Schlüssel, der von Blue- und White-Collar-Beschäftigten gleichermaßen als wichtig angesehen wird, um berufliche Erfolge zu unterstützen (Porubčinová 2015). Dies kann auch die Akzeptanz bei der Einführung neuer Technologien fördern und mit Digitalisierung verbundene Ängste reduzieren (Lager et al. 2019). Das Strukturgleichungsmodell zeigt zudem, das auch der beruflich-sozialen Entkopplung im Zuge betrieblicher Veränderungen frühzeitig begegnet und bei drohender Verunsicherung der Beschäftigten Maßnahmen zur beruflichen Bindung und Integration angestoßen werden sollten. Hier kann die Digitalisierung sogar unterstützen: Gerade in Situationen wie z.B. der CoronaPandemie, bei der Beschäftigte verstärkt daheim arbeiten (Pauly und Holdampf-Wendel 2020) und sich von ihren beruflich-sozialen Strukturen zu entkoppeln und gar zu vereinsamen drohen (Rauner 2020) und womöglich beruflich verunsichert sind, können unterstützende digitale Systeme zur Stärkung der Selbstwirksamkeit beitragen, was wiederrum mit höherer allgemeiner Veränderungsbereitschaft assoziiert ist und mit geringerer beruflicher Unsicherheit einhergeht. Daher bedarf es im Betrieben aufeinander abgestimmter Maßnahmen, um gleichsam sowohl technische und organisatorische, als auch personenbezogene und sozial-integrative Aspekte im Zuge der Digitalisierung zu fördern.

Funding Open Access funding provided by Projekt DEAL.

Open Access Dieser Artikel wird unter der Creative Commons Namensnennung 4.0 International Lizenz veröffentlicht, welche die Nutzung, Vervielfältigung, Bearbeitung, Verbreitung und Wiedergabe in jeglichem Medium und Format erlaubt, sofern Sie den/die ursprünglichen Autor(en) und die Quelle ordnungsgemäß nennen, einen Link zur Creative Commons Lizenz beifügen und angeben, ob Änderungen vorgenommen wurden.

Die in diesem Artikel enthaltenen Bilder und sonstiges Drittmaterial unterliegen ebenfalls der genannten Creative Commons Lizenz, sofern sich aus der Abbildungslegende nichts anderes ergibt. Sofern das betreffende Material nicht unter der genannten Creative Commons Lizenz steht und die betreffende Handlung nicht nach gesetzlichen Vorschriften erlaubt ist, ist für die oben aufgeführten Weiterverwendungen des Materials die Einwilligung des jeweiligen Rechteinhabers einzuholen.

Weitere Details zur Lizenz entnehmen Sie bitte der Lizenzinformation auf http://creativecommons.org/licenses/by/4.0/deed.de.

\section{Literatur}

Absenger N, Ahlers E, Herzog-Stein A, Lott Y, Maschke M, Schietinger M (2016) Digitalisierung der Arbeitswelt?! Ein Report aus der Hans-Böckler-Stiftung. https://www.econstor.eu/handle/ 10419/175252. Zugegriffen: 8. Okt. 2019

Alder GS, Schminke M, Noel TW, Kuenzi M (2008) Employee reactions to internet monitoring: the moderating role of ethical orientation. J Bus Ethics 80(3):481-498
Atanasoff L, Venable MA (2017) Technostress: Implications for adults in the workforce. Career Dev Q 65(4):326-338

Bakker AB, Demerouti E (2007) The job demands-resources model: state of the art. J Manag Psychol 22(3):309-328

Bakker AB, Demerouti E (2014) Job demands-resources theory. In: Cooper C, Chen P (Hrsg) Wellbeing: A complete reference guide. Wiley-Blackwell, Chichester, S 37-64

Bandura A (1982) Self-efficacy mechanism in human agency. Am Psychol 37(2):122-147

Bandura A (1997) Self-efficacy: the exercise of control. Freeman, New York

Barber LK, Santuzzi AM (2017) Telepressure and college student employment: The costs of staying connected across social contexts. Stress Health 33(1):14-23

Bayo-Moriones A, Billon M, Lera-López F (2017) Are new work practices applied together with ICT and AMT? Int J Hum Res Manag 8(4):553-580

Becke G (2018) Soziale Zugehörigkeit - eine fragile organisationale Ressource bei digitaler und vermarktlichter Arbeit. In: Geramanis O, Hutmacher S (Hrsg) Identität in der modernen Arbeitswelt. Springer Gabler, Wiesbaden, S 267-281

Berg C (2017) Zusammenhänge zwischen beruflicher Identität, Commitment und Arbeitszufriedenheit. Z Arb Wiss 71(3):169-178

BMAS (2016) Monitor-Digitalisierung am Arbeitsplatz: Aktuelle Ergebnisse einer Betriebs- und Beschäftigtenbefragung. http://www. bmas.de/SharedDocs/Downloads/DE/PDF-Publikationen/a875monitor-digitalisierung-am-arbeitsplatz.pdf?_blob=publication File\&v=2. Zugegriffen: 3. Juli 2019

BMWi (2018) Monitoring-Report Wirtschaft DIGITAL. https://www. bmwi.de/Redaktion/DE/Publikationen/Digitale-Welt/monitoringreport-wirtschaft-digital-2018-langfassung.pdf?_blob= publicationFile\&v=12. Zugegriffen: 3. Juli 2019

Carter WR, Nesbit PL, Badham RJ, Parker SK, Sung LK (2018) The effects of employee engagement and self-efficacy on job performance: a longitudinal field study. Int J Hum Res Manag 29(17):2483-2502

Clauß E, Verworn B (2019) Möglichkeiten 4.0: Chancen der Digitalisierung für Beschäftigte und Unternehmen. In: Badura B, Ducki A, Schröder H, Klose J, Meyer M (Hrsg) Krise und Gesundheit - Ursachen, Prävention, Bewältigung. Fehlzeiten-Report 2017. Springer, Berlin, S 51-61

Demerouti E, Nachreiner F (2019) Zum Arbeitsanforderungen-Arbeitsressourcen-Modell von Burnout und Arbeitsengagement Stand der Forschung. Z Arb Wiss 73(2):119-130

Dengler K, Matthes B, Wydra-Somaggio G (2018) Digitalisierung in den Bundesländern: Regionale Branchen- und Berufsstrukturen prägen die Substituierbarkeitspotenziale. IAB, Nürnberg

Van der Doef M, Maes S (1998) The job demand-control (-support) model and physical health outcomes: a review of the strain and buffer hypotheses. Psychol Health 13(5):909-936

Dooley D (2003) Unemployment, underemployment, and mental health: Conceptualizing employment status as a continuum. Am J Community Psychol 32(1-2):9-20

Ferris DL, Brown DJ, Berry JW, Lian H (2008) The development and validation of the Workplace Ostracism Scale. J Appl Psychol 93(6):1348-1366

Geiser C (2011) Lineare Strukturgleichungsmodelle. In: Geiser C (Hrsg) Datenanalyse mit Mplus. Eine anwendungsorientierte Einführung. Springer, Wiesbaden, S 41-92

Gerten E, Beckmann M, Bellmann L (2018) Controlling working crowds: The impact of digitalization on worker autonomy and monitoring across hierarchical levels. Center of Business and Economics, Basel

Grawitch MJ, Werth PM, Palmer SN, Erb KR, Lavigne KN (2018) Self-imposed pressure or organizational norms? Further examination of the construct of workplace telepressure. Stress Health 34(2):306-319 
Hackman J, Oldham G (1980) Work redesign. Addison-Wesley, Massachusetts

Hämmerle M, Rally P, Scholtz O (2017) Digitalisierung und Arbeitswelt in Chemie und Pharma Baden-Württemberg. Fraunhofer-Institut für Arbeitswirtschaft und Organisation IAO, Stuttgart

Härtwig C, Sporbert A (2013) Evaluation der Einführung verschiedener Arbeitszeitmodelle. Befunde einer Längsschnittstudie bei der Polizei. Z Arb Wiss 4:243-251

Hasselmann O, Schauerte B, Schröder J (2017) Digitalisierung: Herausforderungen meistern und Krisen vermeiden. Handlungsfelder der Betrieblichen Gesundheitsförderung. In: Badura B, Ducki A, Schröder H, Klose J, Meyer M (Hrsg) Krise und Gesundheit - Ursachen, Prävention, Bewältigung. Fehlzeiten-Report 2017. Springer, Berlin, S 37-52

Heinzer S, Reichenbach R (2013) Die Entwicklung der beruflichen Identität. https://www.ife.uzh.ch/dam/jcr:00000000-272b1a72-0000-00002d44d5b6/Schlussbericht_zum_BBT-Projekt_ Berufliche_Identitaet.pdf. Zugegriffen: 15. Okt. 2018

Hermeier B, Heupel T, Fichtner-Rosada S (Hrsg) (2019) Arbeitswelten der Zukunft. Wie die Digitalisierung unsere Arbeitsplätze und Arbeitsweisen verändert. Springer Gabler, Wiesbaden

Hirsch-Kreinsen H (2016) Digitalisation und Einfacharbeit. FriedrichEbert-Stiftung, Bonn. https://library.fes.de/pdf-files/wiso/12645. pdf. Zugegriffen: 15. Okt. 2018

Hirsch-Kreinsen H, Ittermann P, Niehaus J (Hrsg) (2018) Digitalisierung industrieller Arbeit. Die Vision Industrie 4.0 und ihre sozialen Herausforderungen. Nomos, Baden-Baden

Hoff E-H, Grote S, Dettmer S, Hohner H-U, Olos L (2005) WorkLife-Balance: Berufliche und private Lebensgestaltung von Frauen und Männern in hoch qualifizierten Berufen. Z Arb Organ 49(4):196-207

Jang J, Shin H, Aum H, Kim M, Kim J (2016) Application of experiential locus of control to understand users' judgments toward useful experience. Comput Human Behav 54:326-340

Jerusalem M, Schwarzer R (1986) Selbstwirksamkeit. In: Schwarzer R (Hrsg) Skalen zur Befindlichkeit und Persönlichkeit. Freie Universität Berlin, Berlin, S 15-28

Kubicek B, Paškvan M, Korunka C (2015) Development and validation of an instrument for assessing job demands arising from accelerated change: The intensification of job demands scale (IDS). Eur J Work Organ Psychol 24(6):898-913

Lager H, Delbrügger T, Lenz LT, Roßmann J (2019) Mitarbeiterpartizipation in Zeiten der Digitalisierung mit Building Information Modeling: Gute Praxis digitaler Werkerunterstützung bei der Planung von Fabrikarbeitsstationen. Z Arb Wiss 73(2):229-238

Lempert W (2006) Berufliche Sozialisation und berufliches Lernen. In: Arnold R, Lipsmeier A (Hrsg) Handbuch der Berufsbildung, 2. Aufl. VS, Wiesbaden, S 413-420

Leung L (2011) Effects of ICT connectedness, permeability, flexibility, and negative spillovers on burnout and job and family satisfaction. Hum Technol 7(3):250-267

Loeb C, Stempel C, Isaksson K (2016) Social and emotional self-efficacy at work. Scand J Psychol 57(2):152-161

Maddux JE (2016) Self-efficacy. In: Trusz S, Babel P (Hrsg) Interpersonal and intrapersonal expectancies. Routledge, London, S $41-46$

Maier GW, Engels G, Steffen E (Hrsg) (2017) Handbuch Gestaltung digitaler und vernetzter Arbeitswelten. Springer, Berlin

Martin K, Freeman RE (2003) Some problems with employee monitoring. J Bus Ethics 43(4):353-361

Mellner C (2016) After-hours availability expectations, work-related smartphone use during leisure, and psychological detachment: The moderating role of boundary control. Int J Workplace Health Manag 9(2):146-164

Metz A, Rothe H (2017) Screening psychischer Arbeitsbelastung. Ein Verfahren zur Gefährdungsbeurteilung. Springer, Wiesbaden

Mohr C, Duresso R (2012) Erwerblosigkeit. In: Bamberg E, Mohr G, Busch C (Hrsg) Arbeitspsychologie. Hogrefe, Göttingen, S 187-204
Mohr G, Rigotti T, Müller A (2007) Irritations-Skala zur Erfassung arbeitsbezogener Beanspruchung (IS). Hogrefe, Göttingen

Moos DC, Azevedo R (2009) Learning with computer-based learning environments: A literature review of computer self-efficacy. Rev Educ Res 79(2):576-600

Ninaus K, Diehl S, Terlutter R, Chan K, Huang A (2015) Benefits and stressors-Perceived effects of ICT use on employee health and work stress: An exploratory study from Austria and Hong Kong. Int J of Qualitative Studies on Health and Well-Being 10(1). https://www.tandfonline.com/doi/pdf/10.3402/qhw.v10.28838? needAccess=true. Zugegriffen: 9. Juli 2019

Pauly B, Holdampf-Wendel A (2020) Corona-Pandemie: Arbeit im Homeoffice nimmt deutlich zu. https://www.bitkom.org/Presse/ Presseinformation/Corona-Pandemie-Arbeit-im-Homeofficenimmt-deutlich-zu. Zugegriffen: 20. März 2020

Porubčinová M (2015) Work competencies of white and blue collar workers in the context of information society development. Progn Práce 7(2):115-133

Priddat BP, West K-W (2016) Digitale Wirtschaft. Mit besonderem Blick auf die chemische Industrie. www.cssa-wiesbaden.de/ fileadmin/Bilder/B\%C3\%BCcher_Brosch\%C3\%BCren/Paperscssa/cssa-paper_digitale_Wirtschaft_1_2016.pdf. Zugegriffen: 23. März 2018

Rauner M (2020) Das ist für uns wie ein natürliches Großexperiment. Interview. https://www.zeit.de/wissen/gesundheit/2020-03/ einsamkeit-coronavirus-isolation-ausgangssperre-psychologiehomeoffice/komplettansicht?print. Zugegriffen: 23. März 2020

Rusch S (2019) Stressmanagement. Ein Arbeitsbuch für die Aus-, Fortund Weiterbildung. (2. Aufl.). Berlin: Springer

Salanova M, Cifre E, Llorens S, Martínez I, Lorente L (2011) Psychosocial risks and positive factors among construction workers. In: Cooper C, Burke R, Clarke S (Hrsg) Occupational health and safety: Psychological and behavioral aspects of risk. Gower, Aldershot, S 295-320

Schafer JL, Graham JW (2002) Missing data: our view of the state of the art. Psychol Methods 7(2):147-177

Schobert DB (2007) Grundlagen zum Verständnis von Work-Life-Balance. In: Esslinger AS, Schobert DB (Hrsg) Erfolgreiche Umsetzung von Work-Life Balance in Organisationen. DUV, Wiesbaden, S 19-33

Sellberg C, Susi T (2014) Technostress in the office: a distributed cognition perspective on human-technology interaction. Cogn Technol Work 16(2):187-201

Shoji K, Cieslak R, Smoktunowicz E, Rogala A, Benight CC, Luszczynska A (2016) Associations between job burnout and self-efficacy: a meta-analysis. Anxiety Stress Coping 29(4):367-386

Sonnentag S, Kruel U (2006) Psychological detachment from work during off-job time: the role of job stressors, job involvement, and recovery-related self-efficacy. Eur J Work Organ Psychol 15(2):197-217

Spreitzer GM (1995) Psychological empowerment in the workplace: dimensions, measurement, and validation. Acad Manag J 38(5):1442-1465

Stanton JM, Weiss EM (2000) Electronic monitoring in their own words: an exploratory study of employees' experiences with new types of surveillance. Comput Human Behav 16(4):423-440

Statistisches Bundesamt (Destatis) (2017) Unternehmen, Beschäftigte und Umsatz nach Wirtschaftsabschnitten. https://www. destatis.de/DE/Themen/Branchen-Unternehmen/Unternehmen/ Unternehmensregister/Tabellen/unternehmen-beschaeftigte-umsatzwz08.html. Zugegriffen: 23. Juli 2019

Statistisches Bundesamt (Destatis) (2019) Anzahl der jährlich versendeten E-Mails (ohne Spam) in Deutschland in den Jahren 2000 bis 2018 (in Milliarden). https://de.statista.com/statistik/ daten/studie/392576/umfrage/anzahl-der-versendeten-e-mailsin-deutschland-pro-jahr/. Zugegriffen: 1. Okt. 2019

Stawarz K, Cox AL, Bird J, Benedyk R (2013) I'd sit at home and do work emails: how tablets affect the work-life balance of of- 
fice workers. Human Factors Konferenz, Paris (Vortrag). http:// discovery.ucl.ac.uk/1396455/1/StawarzCoxBirdBenedyk_worklife balance.pdf. Zugegriffen: 8. Okt. 2019

Stegmann S, van Dick R, Ullrich J, Charalambous J, Menzel B, Egold N, Wu TT (2010) Der work design questionnaire. Z Arbeits Organisationspsychol 54:1-28

Syrek C, Bauer-Emmel C, Antoni C, Klusemann J (2011) Entwicklung und Validierung der Trierer Kurzskala zur Messung von WorkLife Balance (TKS-WLB). Diagnostica 57(3):134-145

Szebel A (2015) Veränderungskompetenz von Mitarbeitern. Eine empirische Untersuchung zur differentiellen Konstrukterschließung individuellen Veränderungskompetenz von Mitarbeitern unter besonderer Berücksichtigung des Einflusses dispositionaler Persönlichkeitsfaktoren. Dissertation. Universität Köln, Köln

Tarafdar M (2018) Mobile, Remote and Blue-Collar: Using Information and Communication Technology to Elevate Workplace WellBeing. 26. Europäische Konferenz der Informationstechnologi- en, Portsmouth (Vortrag). http://ecis2018.eu/wp-content/uploads/ 2018/09/1372-doc.pdf. Zugegriffen: 9. Juli 2019

Ventura M, Salanova M, Llorens S (2015) Professional self-efficacy as a predictor of burnout and engagement: The role of challenge and hindrance demands. J Psychol 149(3):277-302

Windelband L (2019) Arbeiten und Lernen in einer zunehmend digitalisierten Arbeitswelt. In: Badura B, Ducki A, Schröder H, Klose J, Meyer M (Hrsg) Fehlzeiten-Report 2019. Digitalisierung gesundes Arbeiten ermöglichen. Springer, Berlin, S 40-49

Windelband L, Spöttl G (2018) Qualifikationsstruktur und Kompetenzprofile von Fachkräften im aktuellen ökonomisch-technischen Wandel. In: Arnold R, Lipsmeier A, Rohs M (Hrsg) Handbuch Berufsbildung. Springer, Wiesbaden, S 1-14

Zika G, Helmrich R, Maier T, Weber E, Wolter MI (2018) Regionale Branchenstruktur spielt eine wichtige Rolle: Arbeitsmarkteffekte der Digitalisierung bis 2035. IAB, Nürnberg 